\title{
The intermediate band approach in the third solar cell generation context
}

\author{
Germán González-Díaz - I. Mártil , A. del Prado , D. Pastor ‘, J. Olea , E. García-Hemme , R. García- \\ Hernansanz , P. Wahnón
}

\begin{abstract}
Within the framework of the third solar cell generation some new ideas to enlarge the spectral response of the solar cells toward the infrared have been proposed. Among them the inclusion of an Intermediate Band (IB) seems to be very promising. This paper will deal with one of the ways to generate the IB namely the deep level center approach. We will discuss not only its existence but also the carriers lifetime recovery which is necessary to obtain the expected increase of the solar cell efficiency.
\end{abstract}

Keywords-Titanium, vanadium, silicon, ion implantation, pulsed laser melting, intermediate band, solar cells.

\section{INTRODUCTION}

It is well known that the introduction of deep levels in a semiconductor produces a deleterious effect in the carrier lifetime which has been positively used to control the switching time in commutation devices. Apart from this effect, the deep levels have to be avoided in usual technologies and specially in solar cells due to the detrimental effect in the efficiency.

Prof. Luque and coworkers (1) suggested in 1997 that if deep levels could be introduced in enough quantity to surpass the Mott level, that is to say the transition metal - insulator, a new band could be created. This new band or Intermediate Band (IB) can be used as a stepping stone for photons with energy lower than band gap to promote electrons from the valence band to conduction band. The incorporation of one of this IB to a standard solar cell will be one of the factors that could bring to an improvement in its efficiency.

To obtain the maximum efficiency it is clear that the carrier concentration generated in the steady state by the solar illumination has to be maximized, which makes it necessary to reach the maximum value of the carrier lifetime. Shockley Read Hall theory predicts a dramatic decrease in lifetime but in the case of an IB is formed Luque foresees a lifetime recovery, based on the configuration diagrams theory (2).

This point of lifetime recovery has been an object of controversy $(3,4)$, as it has been argued through theoretical calculation that to obtain this effect the IB energetic bandwidth has to be broad enough to reach one of the "normal" bands of the semiconductor namely the conduction or the valence band. In this case all the advantages of the IB disappear and we will obtain a new semiconductor with a smaller band gap.

Some groups (5) have calculated the optimum semiconductor gap and the energetic impurity level for a maximum efficiency solar cell, but up to now and based on this technology the IB has been only obtained in $\mathrm{Si}$.

Besides it application to solar cells, the IB could be used easily to build infrared detectors. Important efforts (6) are been carried out to obtain silicon based infrared detectors, mainly supersaturating Si wafers with chalcogenides. This field has a strategic interest and could benefit from the very mature silicon technology.

From a theoretical point of view P. Wahnon et al (7) has proved the possibility of having a half filled band when $\mathrm{Ti}$ is incorporated into $\mathrm{Si}$. Ti produces a deep level and is well known as a carrier lifetime killer.

From the experimental point of view the main difficulty is the incorporation in the silicon lattice of the required deep level concentration. Most of the transition metals if not all have solid solubility orders of magnitude lower than Mott limit and this fact precludes all the equilibrium processes. Supersaturated concentrations could be obtained using non equilibrium techniques as Ion Implantation (II) and Pulsed Laser Melting (PLM).

\section{EXPERIMENTAL AND CHARACTERIZATION}

Samples $1 \times 1 \mathrm{~cm}^{2}$ in size of n-type Si (111) with a thickness of $300 \mu \mathrm{m}\left(\rho \approx 200 \Omega \mathrm{cm} ; \mu \approx 1500 \mathrm{~cm}^{2} \mathrm{~V}^{-1} \mathrm{~s}^{-1}, \mathrm{n} \approx 2.2 \times 10^{13} \mathrm{~cm}^{-3}\right.$ at room temperature) were implanted at $32 \mathrm{keV}$ with different transition metals at a doses from $10^{13}-10^{16} \mathrm{~cm}^{-2}$. Subsequently the implanted samples were PLM processed at energies from $0.6-1.4 \mathrm{~J} / \mathrm{cm}^{2}$ with a $\mathrm{KrF}$ excimer laser $(248 \mathrm{~nm})$ at IPG Photonics. Also, some Si wafer without implantation and $\mathrm{Si}$ samples implanted with Si at $170 \mathrm{keV}$ and $10^{16} \mathrm{~cm}^{-2}$ were processed by PLM at $1 \mathrm{~J} / \mathrm{cm}^{-2}$ for comparatives purposes.

Implanted samples have been characterized by ToF-SIMS, TEM, (8) Raman (9) and RBS (10). Figure 1 shows the TEM images and the SIMS profiles for Ti implantation at various doses. It can be seen that at doses of $10^{15} \mathrm{~cm}^{-2}$ the crystallinity has been fully recovered. For doses until $10^{16} \mathrm{~cm}^{-2}$ the annealing is not perfect and the resulting implanted layer 
becomes policrystalline. Same results hold for Raman spectroscopy. RBS determines clearly that $\mathrm{Ti}$ is located in interstitial places. Only a few percent is located in substitutional positions.

Electrical characterization was made at variable temperature $(10-300 \mathrm{~K})$ placing the samples inside a closedcycle Janis cryostat. In order to avoid moisture condensation, the cryostat was attached to a vacuum pump. A Keithley SCS 4200 model with four source and measure units was used to perform the sheet resistance and Hall effect measurements with the van der Pauw configuration. A Kepco bipolar current source was used to feed an electromagnet.

Figures $2 \mathrm{a}$ and $2 \mathrm{~b}$ represent the sheet resistance and the mobility of a series of Ti implantation with doses from $10^{14}$ to $10^{16} \mathrm{~cm}^{-2}$ and annealed at $0.8 \mathrm{~J} / \mathrm{cm}^{2}$. Also the substrate resistivity and mobility is represented in both plots. As it can be seen, the electrical properties of the $10^{14} \mathrm{~cm}^{-2}$ sample are indistinguishable from those of the substrate. Nevertheless sheet resistance for the other doses presents an unconventional behavior. It should be expected that a parallel structure as the one we have should have a sheet resistance lower than that of each one of its branches. In the figure 2a) sheet resistance of

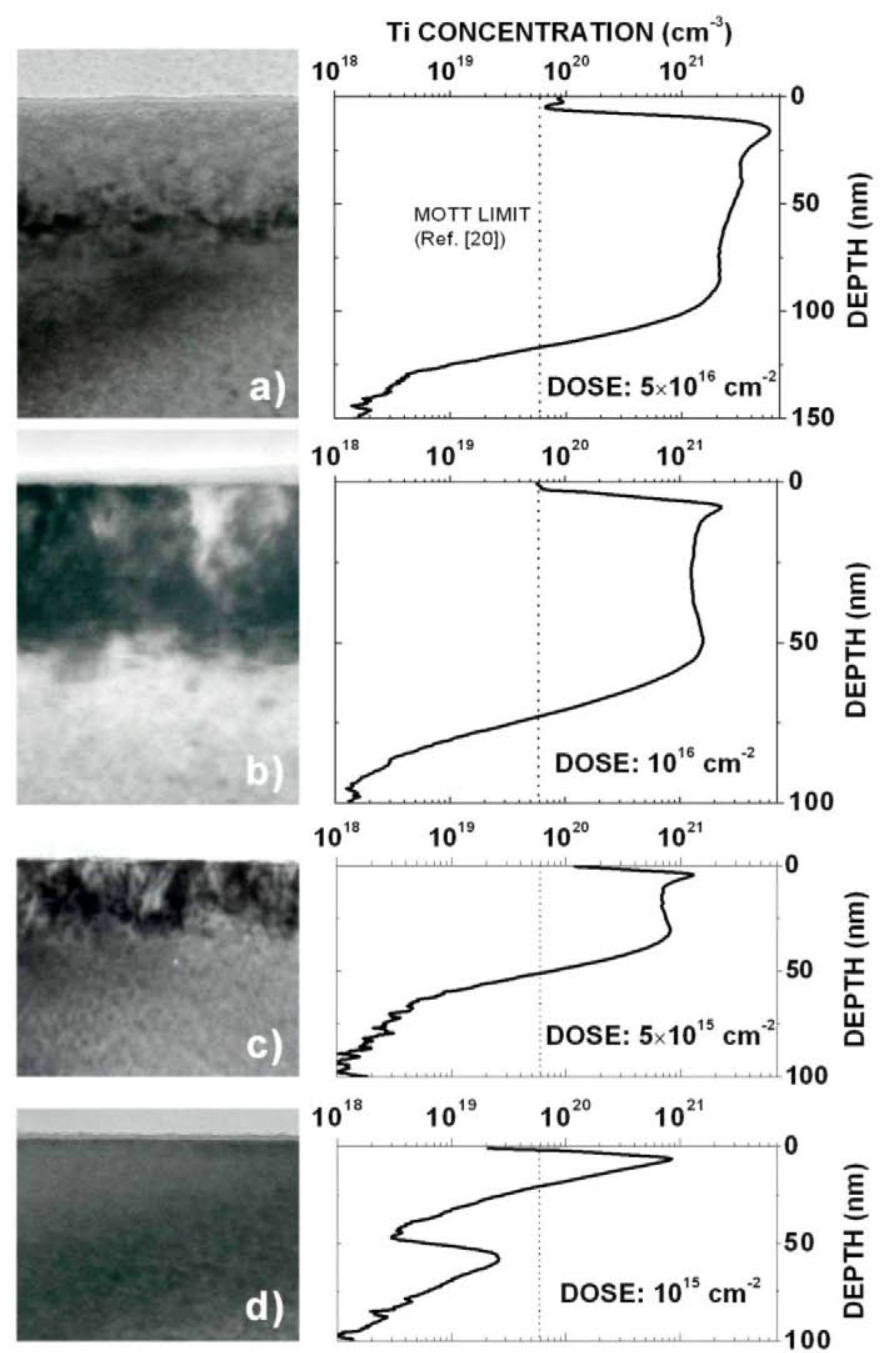

Figure. 1. TEM images and ToF-SIMS profiles of the layers PLM annealed at $0.8 \mathrm{~J} / \mathrm{cm} 2$ for all the implanted doses. (a) Dose: $5 \times 10^{16}$ $\mathrm{cm}^{-2}$, (b) dose: $10^{16} \mathrm{~cm}^{-2}$, (c) dose: $5 \times 10^{15} \mathrm{~cm}^{-2}$ and (d) dose: $10^{15} \mathrm{~cm}^{-2}$. the whole sample crosses the sheet resistance of the substrate. Also the behavior of mobility is uncommon.

These behaviors have been simulated with ATLAS framework and also an analytical model has been developed. The model is sketched in fig 3. A current limitation between the implanted layer and the substrate is assumed and it is represented in the picture as Rt. The van der Paw current I is divided between both layers in inverse proportion to the resistances and each layer generates the van der Paw voltage at the opposite terminals. The differential voltmeter reads a voltage which takes into account the resistance seen between the contacts in each layer.

Solving the circuit we can obtain the following equations for the sheet resistance and mobility:

$$
\begin{array}{r}
R_{\text {sheet }}=\frac{G_{C 1}+G_{C 2} F^{2}}{\alpha\left(G_{C 1}+G_{C 2} F\right)^{2}} \\
\mu_{\text {eff }}=\frac{\mu_{1} G_{C 1}+\mu_{2} G_{C 2} F^{2}}{G_{C 1}+G_{C 2} F^{2}}
\end{array}
$$

Where $F=G_{t} /\left(G_{t}+G_{C 2}\right), \mathrm{Gc}_{1}$ and $\mathrm{Gc}_{2}$ are the sheet conductance of the layer 1 and layer 2 respectively, Gt is the transverse or limiting conductance and $\alpha$ is a geometric factor that takes into
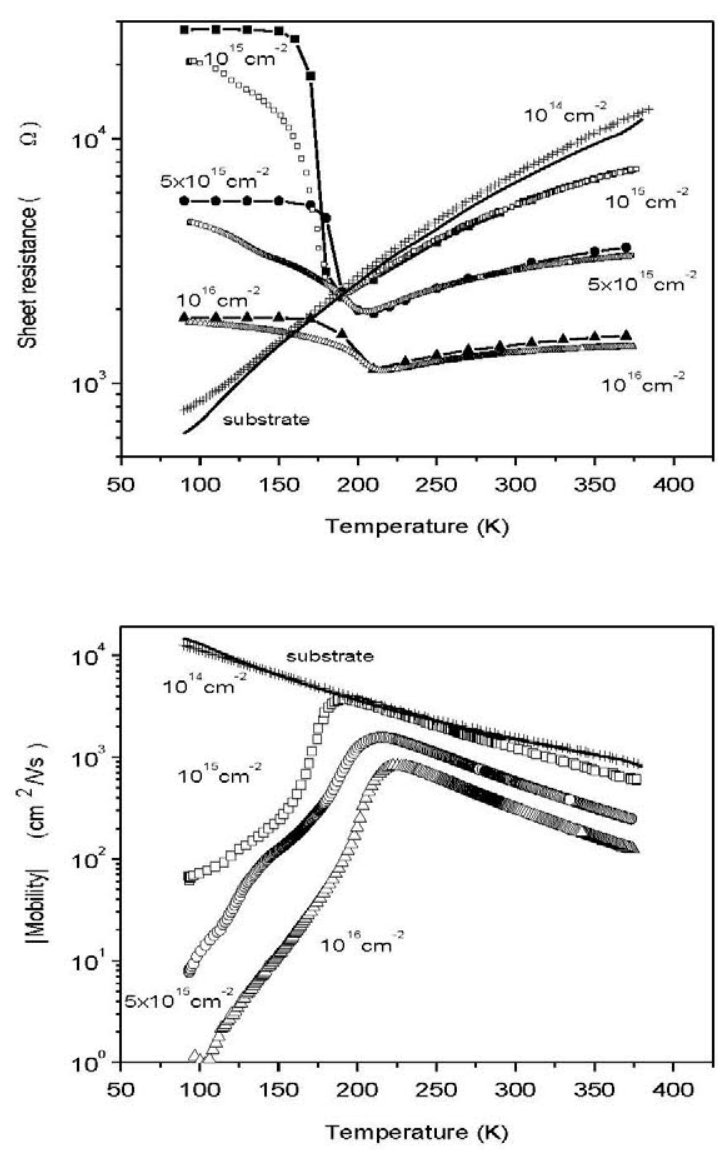

Figure 2: a) Sheet resistance as a function of measured temperature for a $\mathrm{n}-\mathrm{Si}$ substrate $(-)$ and for double sheet TIL/n-Si substrate for different implantation doses: $10^{14} \mathrm{~cm}^{-2}\left(+\right.$ experimental); $10^{15} \mathrm{~cm}^{-2}(\square$ experimental; a ATLAS simulation); $5 \times 10^{15} \mathrm{~cm}^{-2}$ (o experimental; - ATLAS simulation) and $10^{16} \mathrm{~cm}^{-2}$ ( $\Delta$ experimental; $\boldsymbol{\Delta}$ ATLAS simulation) b) Mobility absolute value as a function of the measured temperature for a $\mathrm{n}-\mathrm{Si}$ substrate $(-)$ and for double sheet TIL/n-Si substrate for different implantation doses: $10^{14} \mathrm{~cm}^{-2}(+) ; 10^{15} \mathrm{~cm}^{-2}(\square)$; $5 \times 10^{15} \mathrm{~cm}^{-2}(0)$ and $10^{16} \mathrm{~cm}^{-2}(\Delta)$. 


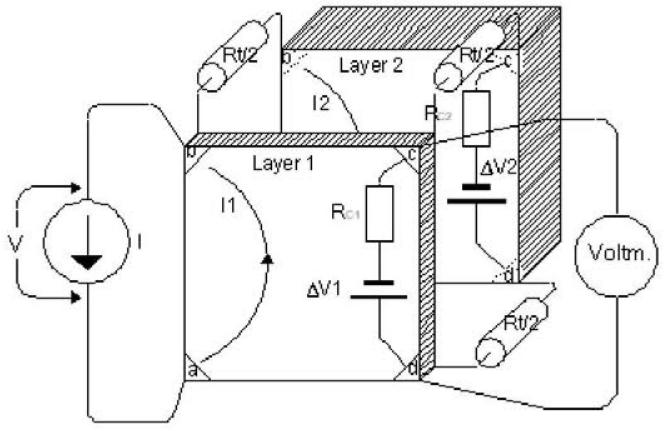

Figure 3: Schematic 3D view of the van der Pauw set-up account the relative size between the contacts and the substrate size.

Same procedure was used with Vanadium implanted silicon. Fig 4 and 5 presents a comparison between the sheet

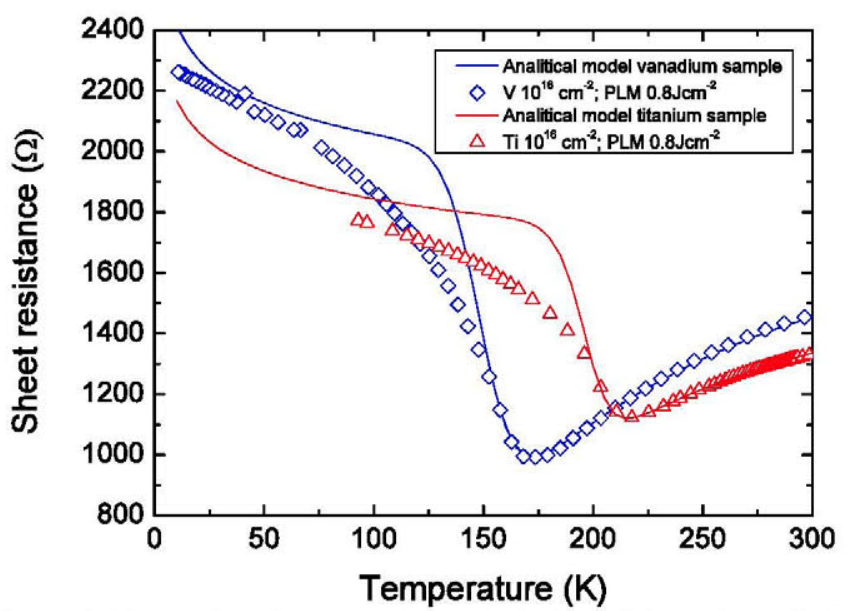

Figure 4 Comparison between the analytical model results and the measured values for the sheet resistance as a function of the temperature in the Ti implanted sample at $10^{16} \mathrm{~cm}^{-2}$ dose and the $\mathrm{V}$ implanted sample at $10^{16} \mathrm{~cm}^{-2}$ dose subsequently PLM processed at $0.8 \mathrm{Jcm}^{-2}$

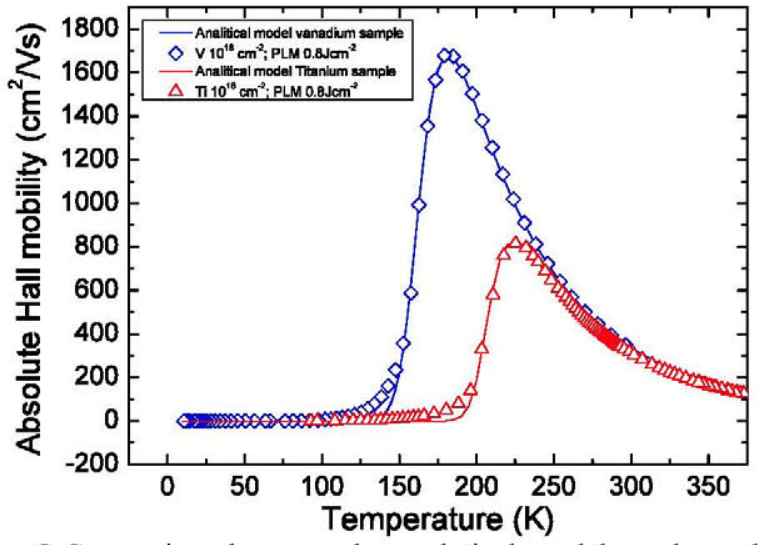

Figure 5 Comparison between the analytical model results and the measured values for the mobility as a function of the temperature in the Ti implanted sample at $10^{16} \mathrm{~cm}^{-2}$ dose and the $\mathrm{V}$ implanted sample at $10^{16} \mathrm{~cm}^{-2}$ dose subsequently PLM processed at $0.8 \mathrm{Jcm}^{-2}$. resistance and the mobility of the $\mathrm{V}$ and Ti showing the shame shape in both variables but with differences in the temperature for the minimum on the resistivity and for the maximum in the mobility.

An important feature of the mobility is the change of its sign at low temperatures that happens for all the doses and all the PLM energies. Figure 6 presents this change for a $10^{16} \mathrm{~cm}^{-2}$ Ti sample annealed at $0.8 \mathrm{~J} / \mathrm{cm} 2$. All the samples were $\mathrm{N}$ type for high temperature changing to $\mathrm{P}$ type at low temperatures.

The model used gives us the carrier density on the IB and its mobility. It is important to note that this density is almost constant until the minimum obtainable temperature in our cryostat, namely $7 \mathrm{~K}$. This semimetal behavior together with the low mobility $\mathrm{p}$ type defines clearly a half filled band which is not compatible with a conduction process based on isolated defects. The fact to have the sheet resistance minimum at different temperatures depending on the implanted element is also another argument indicating the IB formation

The former arguments prove the existence of the IB. However an IB without a recovery in the carrier lifetime is intrinsically useless. This recovery has been proved in (11) and (12) by measuring the photoconductive response. Figure 7 presents the spectral photoconductance normalized to the incident photon flux of a series of Ti implantations on a $3000 \Omega . \mathrm{cm}$ substrate. The infrared substrate response is also plotted. In this last case the excess carriers are due to the generation on the superficial states combined with the high carrier lifetime typical of high resistance substrates (13).

Samples implanted at $10^{13} \mathrm{~cm}^{-2}$ shows a dramatic decrease in the photoconductivity which is related to the presence on the silicon surface of a high quantity of deep levels. The Ti concentration for this dose is lower than the Mott limit and consequently they will have a very important recombination velocity. Contrarily as it can be assumed, an increment on the Ti concentration produces higher photoconductivity, i.e. a higher excess carrier concentration. Even with a dose of $10^{15} \mathrm{~cm}^{-2}$ we obtain higher photoconductivity than the substrate itself though in in a limited energy range. That means that not only we have recovered the mean free path of the electrons in

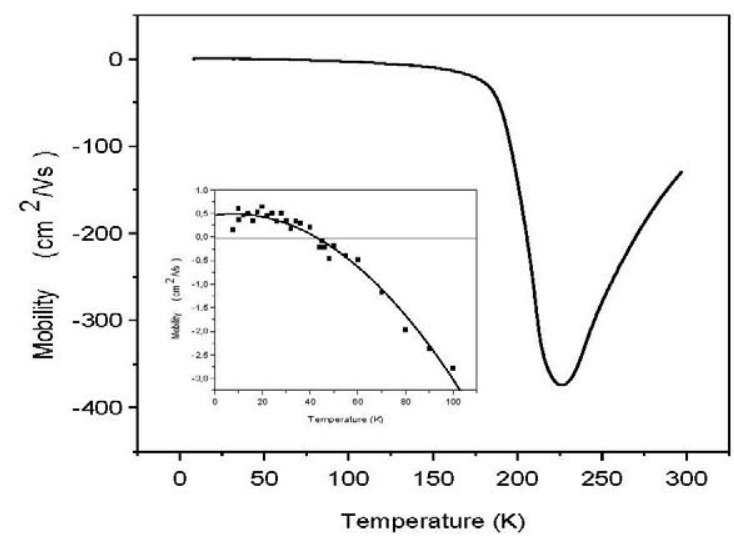

Figure 6: Hall mobility for a sample implanted with a dose of $10^{16} \mathrm{~cm}^{-2}$ and PLM at $0.8 \mathrm{~J} / \mathrm{cm}^{2}$ measured down to $7 \mathrm{~K}$ and showing p-type conductivity at low temperatures. Inset shows an enlargement of the mobility at low temperature. 
the substrate because the recombination in the implanted zone has decreased but that the implanted layer is giving carriers to

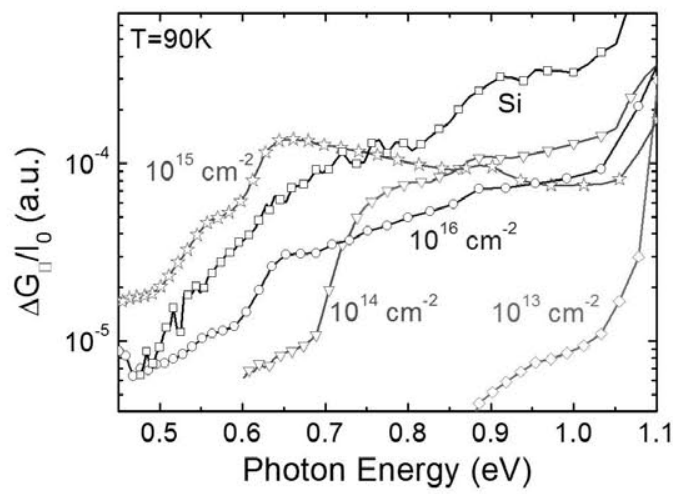

Fig 7: Normalized sheet photoconductance versus photon energy for Ti implanted samples with doses from 1014 - $1016 \mathrm{~cm}-2$. Also shown $3000 \Omega . \mathrm{cm}$ substrate sheet photoconductance

the substrate.

Figure 8 shows the sheet photoconductance for a $200 \Omega \mathrm{cm}$ substrate and for the same substrate implanted with $10^{16} \mathrm{~cm}^{-2} \mathrm{~V}$. As the substrate conductivity is now lower than the previous case the increase in photoconductance is due to the implanted V. It is important to note the very sharp photoconductance front at about $0.2 \mathrm{eV}$. Also the coincidence for the implanted and non implanted sample for energies higher than the fundamental $\mathrm{Si}$ absorption

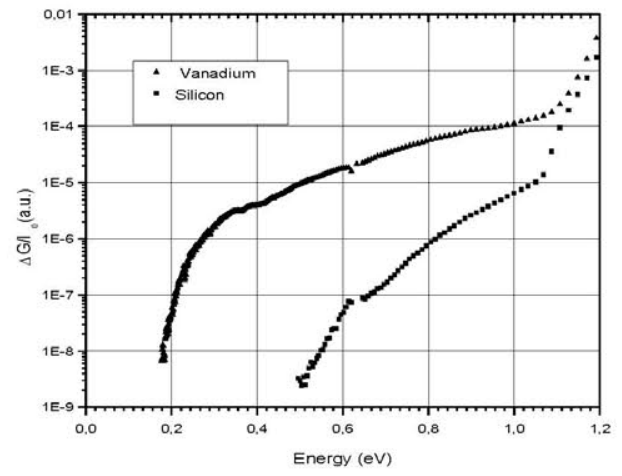

Fig 8: Sheet photoconductance for a V implanted sample and for the $200 \Omega$ substrate

\section{CONCLUSIONS}

We present a model describing the electrical behaviour of $\mathrm{V}$ and $\mathrm{Ti}$ implanted layers on $\mathrm{Si}$ substrates. These implantations have been analysed using a variety of experimental techniques to determine the position of the impurity in lattice, the degree of cristallinity and so on. We conclude that a IB is formed and we develop an electrical model wich allow us to know the carrier concentration and the mobility of the IB.
Photoconductance measurements confirms the existence of the band and also that the lifetime is recovered being this lifetime higher in more impurified samples unlikely the deep level theory predicts. The photoconductance of $\mathrm{V}$ samples extend down to $0.2 \mathrm{eV}$ having a sharp and very defined front.

\section{ACKNOWLEDGEMENTS}

Authors would like to acknowledge the CAI de Técnicas Físicas of the Universidad Complutense de Madrid for the ion implantations and metallic evaporations. This work was partially supported by the Project NUMANCIA II (Grant No. S-2009/ENE/1477) funded by the Comunidad de Madrid. Research by E. García-Hemme was also supported by a PICATA predoctoral fellowship of the Moncloa Campus of International Excellence (UCM-UPM). J. Olea and D. Pastor thanks Professor A. Martí and Professor A. Luque for useful discussions and guidance and acknowledge financial support from the MICINN within the program Juan de la Cierva (JCI2011-10402 and JCI-2011-11471), under which this research was undertaken.

\section{REFERENCES}

(1) A. Luque and A. Mart_1, Phys. Rev. Lett. 78, 5014-5017 (1997).

(2) Luque, A; Marti, A; Antolin, E; et al. Physica b-Condensed Matter 382-1-2, 320-327 (2006)

(3) Krich, Jacob J.; Halperin, Bertrand I.; Aspuru-Guzik, Alan J.Appl. Phys. 112013707 (2012)

(4) A. Karoui Appl. Phys. Lett. 101, 236101 (2012)

(5) Bremner, S.P.; Levy, M.Y.; Honsberg, C.B. Appl. Phys. Lett. 92 - 17 171110-1-3 (2008)

(6) Persans, Peter D.; Berry, Nathaniel E.; Recht, Daniel; et al. Appl. Phys. Lett. $101-11$ (2012)

(7) Sanchez, K.; Aguilera, I.; Palacios, P.; et al. Phys. Rev. B 79 - 16 (2009)

(8) Olea, J.; Pastor, D.; Toledano-Luque, M.; et al. J. Appl. Phys $1106(2011)$

(9) (9) Pastor, D.; Olea, J.; del Prado, A.; et al. Sem. Science and Technol. $26 \quad 11 \quad$ (2011)

(10) D. Pastor, J. Olea, I. Mártil, G. González Díaz, A. Muñoz and A. Climent Font. J. Appl. Phys 112(2012) 113514

(11) Garcia-Hemme, E; Garcia-Hernansanz, R; Olea, J; Pastor, D; del Prado, A; Martil, I; Gonzalez-Diaz, G Appl. Phys. Lett. 101-19 (2012)

(12) Antolin, E; Marti, A; Olea, J; Pastor, D; Gonzalez-Diaz, G; Martil, I; Luque, A. Appl. Phys. Lett. 94 - 42009

(13) Muller W, Monch W. Phys Rev Lett 255 (1971) 250 